\title{
In Vitro-In Vivo Correlation: Importance of Dissolution in IVIVC
}

\author{
J-M. Cardot ${ }^{1}$, E. Beyssac, and M. Alric \\ Univ. Clermont 1, Biopharmaceutical Department, \\ UFR Pharmacie, 28 Place Henri Dunant, B.P. 38, \\ F-630001 Clermont-Ferrand, France
}

Introduction

orrelations between in vitro and in vivo data (IVIVC) are often used during pharmaceutical development in order to reduce development time and optimize the formulation. A good correlation is a tool for predicting in vivo results based on in vitro data. IVIVC allows dosage form optimization with the fewest possible trials in man, fixes dissolution acceptance criteria, and can be used as a surrogate for further bioequivalence studies; it is also recommended by regulatory authorities (1-5).

Many studies reported in the late ' 70 s and early ' 80 s established the basic concept of IVIVC (6). Various definitions of in vitro-in vivo correlation have been proposed by the International Pharmaceutical Federation (FIP), the USP working group (7), and regulatory authorities such as the FDA or EMEA (2-5). The FDA (2) defines IVIVC as"a predictive mathematical model describing the relationship between an in vitro property of an extended release dosage form (usually the rate or extent of drug dissolution or release) and a relevant in vivo response, e.g., plasma drug concentration or amount of drug absorbed."

As stressed in this definition, IVIVC is more an in vitro-in vivo relationship than a strict correlation. It should be kept in mind that a relationship does not imply a causality link between the in vitro data, in our case, and the in vivo data.

Bases on the type of data used to establish the relationship, three main levels are defined by the FDA:

\section{Level A}

A correlation of this type is generally linear and represents a point-to-point relationship between in vitro dissolution and the in vivo input rate (e.g., the in vivo dissolution of the drug from the dosage form). In a linear correlation, the in vitro dissolution and in vivo input curves may be directly superimposable or may be made to be superimposable by the use of a scaling factor. Nonlinear correlations, while uncommon, may also be appropriate. Alternative approaches to developing a Level A IVIVC are possible.... Whatever the method used to establish a Level A IVIVC, the model should predict the entire in vivo time course from the in vitro data. In this context, the model refers to the relationship between in vitro dissolution of an $E R$ dosage form and an in vivo response such as plasma drug concentration or amount of drug absorbed. e-mail:j-michel.cardot@u-clermont1.fr

\begin{abstract}
Level B
A Level B IVIVC uses the principles of statistical moment analysis. The mean in vitro dissolution time is compared either to the mean residence time or to the mean in vivo dissolution time.... A Level $B$ correlation does not uniquely reflect the actual in vivo plasma level curve, because a number of different in vivo curves will produce similar mean residence time values.
\end{abstract}

\section{Level C}

A Level C IVIVC establishes a single point relationship between a dissolution parameter, for example, $t_{50 \%}$, percent dissolved in 4 hours and a pharmacokinetic parameter (e.g., $A \cup C, C_{\max ,} T_{\max }$ ). A Level $C$ correlation does not reflect the complete shape of the plasma concentration-time curve, which is the critical factor that defines the performance of ER products.

In addition to these three levels, a combination of various levels $C$ is also described:

A multiple Level C correlation relates one or several pharmacokinetic parameters of interest to the amount of drug dissolved at several time points of the dissolution profile.

For the establishment of a correlation as described in the FDA guidance, various parameters can be used as presented in Table 1.

Table 1. Various parameters used in IVIVC depending on the level.

\begin{tabular}{|c|c|c|}
\hline Level & In vitro & In vivo \\
\hline A & Dissolution curve & $\begin{array}{c}\text { Input (absorption) } \\
\text { curves }\end{array}$ \\
\hline B & $\begin{array}{c}\text { Statistical } \\
\text { moments:MDT }\end{array}$ & $\begin{array}{c}\text { Statistical } \\
\text { moments: } \text { MRT, } \\
\text { MAT, etc }\end{array}$ \\
\hline C & $\begin{array}{c}\text { Disintegration } \\
\text { time,Time to have } \\
\text { 10,50,90\% } \\
\text { dissolved, Dissolu- } \\
\text { tion rate, Dissolu- } \\
\text { tion efficiency }\end{array}$ & $\begin{array}{c}\mathrm{C}_{\text {max }} \mathrm{T}_{\text {max }}, \mathrm{K}_{\mathrm{a}}, \text { Time } \\
\text { to have 10,50,90\% } \\
\text { absorbed, AUC } \\
\text { (total or cumula- } \\
\text { tive), }\end{array}$ \\
\hline
\end{tabular}

Level A correlations use all the information of the dissolution and absorption curves, in contrast to levels B or C. The establishment of a relationship implies the use of 
many formulations, each of them giving one pair of data (vitro and vivo). It is obvious that level B or C needs more data and, as they do not use all the information related to vitro and vivo behavior of the formulation, they are less powerful. The FDA ranked the levels as follows:

A Level A IVIVC is considered the most informative and is recommended, if possible. Multiple Level $C$ correlations can be as useful as Level A correlations. However, if a multiple Level $C$ correlation is possible, then a Level A correlation is also likely and is preferred. Level C correlations can be useful in the early stages of formulation development when pilot formulations are being selected. Level B correlations are least useful for regulatory purposes.

In vivo data are obtained from well-standardized fasted studies on healthy volunteers. In attempting to establish a Level A relationship, the major point to consider is the sampling schedule in the "absorption" phase in order to have an accurate representation of the input curve.

Establishing an IVIVC is nothing more complicated than trying to reproduce all the complex phenomena that lead to the in vivo release and solubilisation of the API in the gut in a "simple" in vitro system like a vessel agitated with a paddle! In contrast to in vivo studies, in vitro methods are less "standardized," as USP Apparatus 1 to 4 could be used with various media $(\mathrm{HCl}$, simple buffer, addition of surfactant or enzymes, etc.) and various technical parameters (e.g., volume, rate).

This article will focus on the way to obtain in vitro data.

\section{Data Collection In Vivo Data}

The in vivo data are derived from the plasma concentration curve. Various phenomena can occur after administration of drug depending of the route of administration (8) (Figure 1).

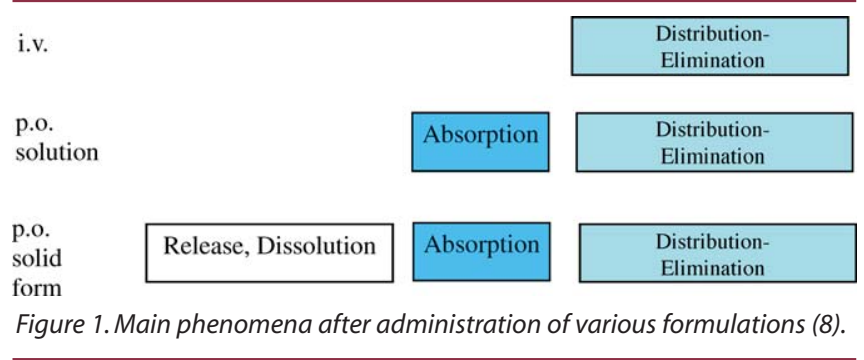

The plasma concentration curve is a global representation; it depends on drug input within the blood, which depends on the dosage form and the properties of the drug, and thereafter its pharmacokinetic input processes. The disposition of the drug afterwards depends only on the drug and patient.

As stated by the FDA (2), absorption curves can be calculated by various methods by estimating "the in vivo absorption or dissolution time course using an appropriate deconvolution technique for each formulation and subject (e.g.,Wagner-Nelson, numerical deconvolution)." After the calculations are performed, the input curve, known as "absorption" curve, is presented as the percentage of the fraction of dose (\%FD:0-100\%) absorbed versus time. This absorption curve represents the sum of all the phenomena such as release or dissolution but also the permeability through the intestinal membranes. To obtain a good estimation of the absorption curves, a sufficient number of well-positioned samples are required.

\section{In Vitro Data}

Interest of In Vitro Dissolution

In the case of oral administration of a solid dosage form, the limiting factor for the appearance of the drug in the blood could be the pure permeability through the intestinal membrane (mainly for Class III and IV of the BCS), the release from the drug dosage form, or the solubility of the active ingredient. In case of solubility-limited product (Class II and IV of BCS), the absorption could be governed by physico-chemical characteristics of the API (such as solubility, dissolution rate, particle size, crystal shape, polymorphism, $\mathrm{pK}_{\mathrm{a}}$, stability in GIT) and not by its permeability or formulation. In the case of release-limited formulations, the absorption is limited by the drug release from the drug dosage form. As described above, to establish IVIVC, the release must be the limiting factor; in this case, solubility (or dissolution rate) and permeability must be faster than the release. Correlations are always possible with Class I drugs and depend on the relative magnitude of the various factors versus the release rate for the other classes (9-14).

The active substance is the core of any formulation; the physico-chemical characteristics and the stability of the API are the main points to be considered first. In the case of oral products, there are two types of formulations. The first is an immediate-release (IR) product that disintegrates and disperses rapidly after administration. The second is an extended-release (ER) formulation, which keeps its integrity throughout much of the GIT (15). In the case of the IR product, the release of both the drug and excipients is

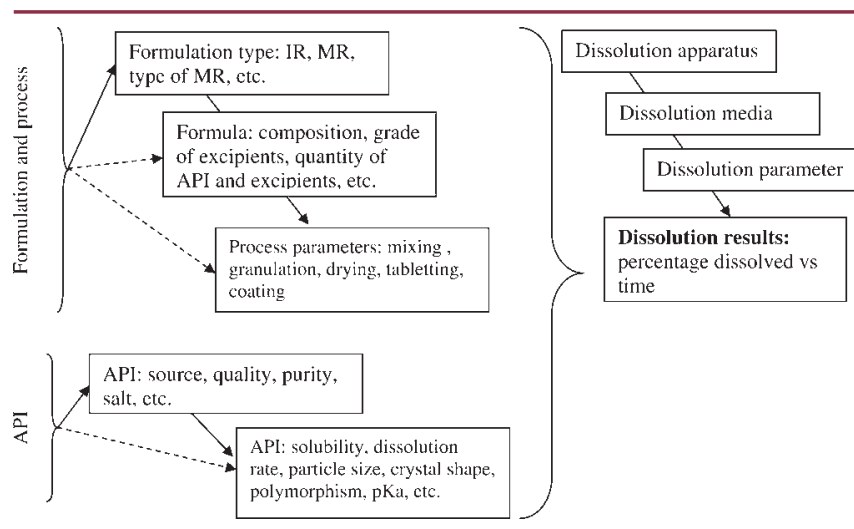

Figure 2. Dissolution as a reflection of the pharmaceutical complexity of the product, full line: directly accessible information, dashed lined: underlined information. 
rapid. The excipients are usually materials without biopharmaceutical properties and do not dramatically modify the release of the drug even if present in large quantity. In the ER case, the excipients are the core of the formulation, and they control the release of drug within the body. For example, the HPMC matrices are biocompatible, do not exhibit any degradation by enzymes and GIT fluids, and have no direct interaction with bile salts, but they interact with water. They swell, form a gel, and then the API slowly diffuses through the formatted gel. Finally, the system is eroded and destroyed after complete gel formation. In this second case, in addition to the characteristics of the API, the characteristics and quantities of excipients and the process itself are of importance.

The release of drug from the dosage form can be studied in vitro, and the dissolution reflects all of the phenomena implied in the formulation (Figure 2).

\section{In Vitro Data}

Various dissolution tests exist and can be used to generate the in vitro data. In order to compare the results, the same method must be used for all the formulations. The in vitro dissolution curves depend on (1) the release characteristics of the formulation and manufacturing process, (2) the characteristics of the API such as the solubility and dissolution rate, and (3) the apparatus, method parameters, dissolution medium (Figure 2).

The dissolution curves are expressed as the percentage dissolved versus time and should usually reach $100 \%$ at infinity (or in practice at the end of dissolution). Based on this curve, various parameters, as presented in Table 1, can be calculated.

The best dissolution method for in vivo-in vitro correlation is, obviously, the method that describes what happens in vivo. The main factors that can influence drug release in vivo are presented in Figure 3. All of these factors cannot be easily reproduced in vitro by a simple dissolution method. In addition, the relative importance of these fac-

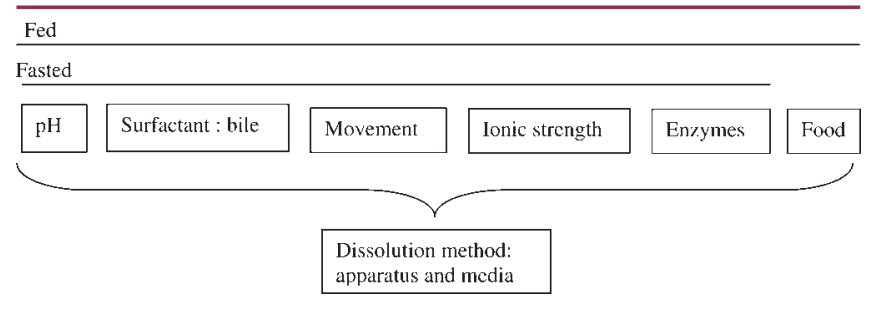

Figure 3. Main factors that can influence the in vivo release, importance for in vitro dissolution method selection.

tors is not similar if the subject is in fed or fasted state; in the fed state, food also has a direct influence on the API or formulation behavior.

\section{In Vitro Media}

Usually, aqueous based media are used, most of them being described in pharmacopeias. To simulate gastric media, the simplest one is composed of $0.1 \mathrm{~N} \mathrm{HCl}$. A more complicated medium called simulated intestine fluid (SIF) can also be used. For other $\mathrm{pHs}$, acetate or phosphate buffers are usually used (or sometime TRIS). For specific applications (e.g., poorly soluble drugs), the pharmacopeias recommend the addition of either enzymes or surfactant. Noncompendial media are also described (16-18) to simulate the fasted and fed states in vivo:fasted state simulated intestinal fluid (FaSSIF) and fed state simulated intestinal fluid (FeSSIF). These media try to reproduce, not the direct food influence on the formulations, but rather the secretions occurring in either fasted or fed state in the small intestine.

\section{In Vitro Apparatus}

Obviously, not all the factors described in Figure 3 can be reproduced in vitro even if some attempts exist. Any compendial method that can discriminate between the formulations may be used, but certain techniques are preferred (i.e., paddle, flow-through cell, basket-USP Apparatus 2,4, and 1 , respectively).

No in vitro system developed to date can maintain the following requirements that correspond to the physiological states in vivo: (1) sequential use of enzymes in physiological amounts, (2) appropriate $\mathrm{pH}$ for the enzymes, (3) removal of the products of digestion, (4) appropriate mixing at each stage of digestion, (5) physiological transit times for each step of digestion, and (6) a peristaltic dynamic approach (19). The TNO Gastro-Intestinal Model (TIM1) is an in vitro system developed at TNO Nutrition and Food Research (Zeist, the Netherlands) that simulates the $\mathrm{Gl}$ tract in man and fulfills the requirements mentioned above. In addition, this in vitro model offers the possibility of introducing a solid meal to investigate all food-drug interactions and food impact on dosage form behaviour. It consists of four serial compartments (Figure 4) simulating the stomach and the three segments of the small intestine: the duodenum, jejunum, and ileum (19-21). Each compartment is filled with water that is pumped from a water bath into the glass jackets around the flexible walls to control the temperature inside the units $\left(37^{\circ} \mathrm{C}\right)$ and the pressure on the flexible walls. Changes in the water pressure enable mixing of the chyme by alternate compression and relaxation of the flexible walls. To control the transit of the chyme, an exponential formula is used for gastric and ileal delivery. Chyme transit is then regulated by opening or closing the peristaltic valves that connect the compartments. The volume in each compartment is monitored by a pressure sensor connected to the computer. The $\mathrm{pH}$ is computer monitored and continuously controlled by secretion of either water or $0.5 \mathrm{M} \mathrm{HCl}(0.25 \mathrm{~mL} / \mathrm{min})$ into the stomach and either electrolytes or $0.5 \mathrm{M} \mathrm{NaHCO}_{3}(0.25$ $\mathrm{mL} / \mathrm{min}$ ) into the small intestine. Simulated gastric $(0.5$ $\mathrm{mL} / \mathrm{min})$, biliary $(0.5 \mathrm{~mL} / \mathrm{min})$, and pancreatic $(0.25$ $\mathrm{mL} / \mathrm{min}$ ) secretions (i.e., pepsin, lipase, pancreatic and bile salts) are introduced into the corresponding compartments by computer-controlled pumps. The model is equipped with hollow-fiber membranes connected to the 


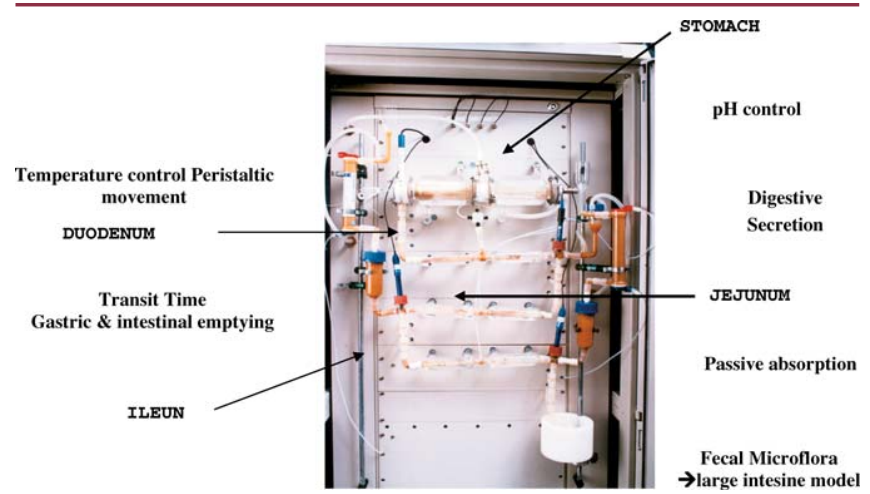

Figure 4. The TIM system.

two compartments representing the jejunum and the ileum, respectively. Water and small molecules are removed from the lumen of the compartments by pumping dialysis fluid $(10 \mathrm{~mL} / \mathrm{min})$ through the hollow fibers. This system mimics GI motility and the mechanical destructive force on drug release; the drug concentration can be measured in jejunal and ileal dialysis fluids, following its passive diffusion through the hollow-fiber membranes connected to the two compartments representing the jejunum and the ileum, respectively.

Although this Artificial Digestive System has been widely used in nutrition studies, few examples of drug dosage forms have been studied. However, this type of system, even if it could be used in drug development with success $(19,22$, $23)$, is not a quality control tool due to its complexity.

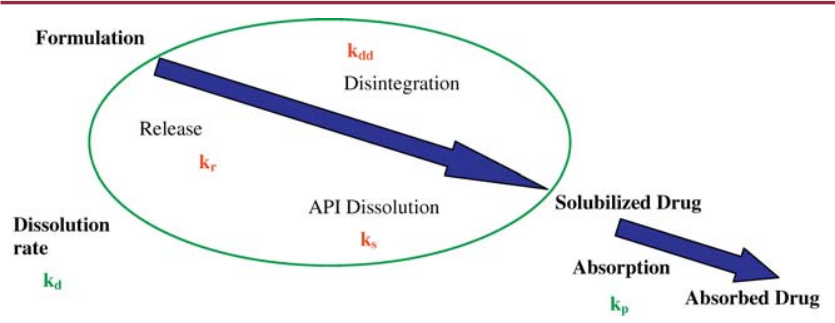

Figure 5. Representation of the main phenomena leading to absorption in vivo, in green apparent observed (macro) phenomena, in red dissolution (micro) phenomena.

The classical methods do not allow all the characteristics of the in vivo interaction to be reproduced, especially the direct food interaction, but can help to study the mean characteristic of drug release. The in vivo drug release can be simplified as a multifactorial process as described in Figure 5.

Three main cases exist for the macro phenomena (green part of the graphic): (1) if $k_{d}$ and $k_{p}$ are fast, drug is well absorbed (class I of the BCS usually), (2) if $k_{d}$ is greater than $\mathrm{k}_{\mathrm{p}}$, the drug is permeation-controlled (Class III), and (3) if $\mathrm{k}_{\mathrm{d}}$ lower than $\mathrm{k}_{\mathrm{p}}$, it is a dissolution-controlled release (Class II).

The various phenomena underlined in $\mathrm{k}_{d}$ (dissolution rate) are of three types, expressed by the micro phenome$\mathrm{na}\left(\mathrm{k}_{\mathrm{r}}, \mathrm{k}_{\mathrm{dd}}\right.$, and $\left.\mathrm{k}_{\mathrm{s}}\right)$ can be split into 4 main categories. First, if $\mathrm{k}_{\mathrm{dd}}$ is greater than $\mathrm{k}_{\mathrm{s}}$, the apparent dissolution is driven by the dissolution of the API. In this case, the study of either the intrinsic dissolution as described in USP and EP or the apparent dissolution determined with the specific USP 4 cell as described in EP is a key factor in dosage form development. Second, if $\mathrm{k}_{\mathrm{dd}}$ is lower than $\mathrm{k}_{\mathrm{s}}$, then the formulation is controlled by disintegration, and the formulation is the key factor. Third, if $k_{d d}$ is close to $k_{s}$ then disintegration is the key factor. Finally, a $\mathrm{k}_{\mathrm{r}}$ that is lower than $\mathrm{k}_{\mathrm{s}}$ indicates slow release, and the critical attribute is the dissolution.

Thus, a classical dissolution technique on the API and the dosage form provides relevant information on the key factors for the establishment of IVIVC, the fact that the release rate of the drug from the drug dosage form is the limiting factor ( $k_{d}$ lower than $k_{p}$, and $k_{r}$ limiting factor of $\left.k_{d}\right)$.

\section{Significance of Level A Correlation}

A Level A correlation defines a linear relationship between in vitro and in vivo data so that measurement of the in vitro dissolution rate alone is sufficient to determine the pharmacokinetic profile in vivo. After a proper validation, IVIVC predicts the in vivo bioavailability results from in vitro dissolution data, and this simulation reflects the in vivo behavior of the various formulations (8).

In the presence of an IVIVC, the FDA states (2),

In vitro dissolution testing is important for (1) providing process control and quality assurance, (2) determining stable release characteristics of the product over time; and (3) facilitating certain regulatory determinations (e.g., absence of effect of minor formulation changes or of change in manufacturing site on performance). In certain cases, especially for ER formulations, the dissolution test can serve not only as a quality control for the manufacturing process but also as an indicator of how the formulation will perform in vivo. Thus, a main objective of developing and evaluating an IVIVC is to establish the dissolution test as a surrogate for human bioequivalence studies....

That highlights the significance of IVIVC and dissolution studies both during development and throughout the life of the product. For example, the establishment of dissolution limits could be based on IVIVC, as stated by FDA. IVIVC can also be used to support biowaivers in two cases over the five categories described in the SUPAC guidance $(2,3)$.

\section{Conclusion}

Level A IVIVCs define the relationship between an in vitro dissolution curve and an in vivo input (absorption) profile. A Level A correlation should always be tried a priori in order to have a tool that allows a complete in vivo prediction from an in vitro dissolution curve and thus accelerates the development and assists in some regulatory aspects (SUPAC). The correlation quality depends solely on the quality of the data. As in vivo data are now well standardized, the main effort must be directed to the in vitro data. Various apparatus and media should be tested and 
assessed in terms of their in vivo predictability. The user should always be aware of the limits of the method and of the confidence of its prediction.

\section{References}

1. International Conference on Harmonisation Steering Committee. Pharmaceutical Development Q8;Draft Consensus Guideline, Released for Consultation at Step 2 of the ICH Process; International Conference on Harmonisation of Technical Requirements for Registration of Pharmaceuticals for Human Use, 18 November 2004.

2. Extended Release Oral Dosage Forms:Development, Evaluation, and Application of In Vitro/In Vivo Correlations; Guidance for Industry; U.S. Department of Health and Human Services, Food and Drug Administration, Center for Drug Evaluation and Research (CDER), U.S. Government Printing Office:Washington, DC, September 1997.

3. Nonsterile Semisolid Dosage Forms Scale-Up and Postapproval Changes: Chemistry, Manufacturing, and Controls; In Vitro Release Testing and In Vivo Bioequivalence Documentation; Guidance for Industry; U.S. Department of Health and Human Services, Food and Drug Administration, Center for Drug Evaluation and Research (CDER), U.S. Government Printing Office: Washington, DC, May 1997.

4. Note for Guidance on Development Pharmaceutics; European Agency for the Evaluation of Medicinal Products, Human Medicines Evaluation Unit, Committee for Proprietary Medicinal Products, CPMP/QWP/155/96; London, U.K., January 1998.

5. Note for Guidance on Modified Release Oral and Transdermal Dosage Forms: Section II (Pharmacokinetic and Clinical Evaluation); European Agency for the Evaluation of Medicinal Products, Human Medicines Evaluation Unit, Committee for Proprietary Medicinal Products, CPMP/EWP/280/96; London, U.K., July 1999.

6. Skelly, J.; Amidon, G.; Barr, W.; Benet, L.; Carter, F.; Robinson, J.; Shah, V.;Yacobi, A. Testing and Correlation for Oral Controlled/Modified-Release Dosage Forms. Pharm. Res. 1990, 7, 975-982.

7. In Vitro and In Vivo Evaluation of Dosage Forms $<1088>$. In United States Pharmacopeia and National Formulary USP 26-NF 21; United States Pharmacopeial Convention, Inc.: Rockville, MD, 2002.

8. Cardot. J-M.;Beyssac, E. In vitro/in vivo correlations:Scientific implications and standardization. Eur.J.Drug Metab. and Pharmacokinet. 1993, 18, 113-120.

9. Amidon, G. L.; Lennernas, H.;Shah, V. P.; Crison, J. R. A theoretical basis for a biopharmaceutic drug classification: the correlation of in vitro drug product dissolution and in vivo bioavailability. Pharm. Res. 1995, 12, 413-420.

10. Dressman, J.;Butler, J; Hempenstall, J.; Reppas, C. The BCS:Where do we go from here? Pharm. Tech. 2001, 25 (7), 68-76.
11. Waiver of In Vivo Bioavailability and Bioequivalence Studies for Immediate-Release Solid Oral Dosage Forms Based on a Biopharmaceutics Classification System; Guidance for Industry; U.S. Department of Health and Human Services, Food and Drug Administration, Center for Drug Evaluation and Research (CDER), U.S. Government Printing Office:Washington, DC, August 2000.

12. Lobenberg, R.; Amidon, G. L. Modern bioavailability and biopharmaceutics classification system. New scientific approaches to international regulatory standards. Eur.J.Pharm. Biopharm. 2000, 50, 3-12.

13. Martinez, M. N.;Amidon, G. L. A mechanistic approach to understanding the factors affecting drug absorption: a review of fundamentals. J.Clin. Pharmacol. 2002, 42,620-643.

14. Wilding, I. R. Evolution of the Biopharmaceutics Classification System (BCS) to oral Modified Release (MR) formulations; what do we need to consider? Eur.J. Pharm.Sci. 1999, 8, 157-159.

15. Abrahamsson, B.;Alpsten, M.;Bake, B.; Larsson, A.;Sjogren, J. In vitro and in vivo erosion of two different hydrophilic gel matrix tablets. Eur.J.Pharm. Biopharm. 1998, 46 (1),69-75.

16. Dressman, J.; Reppas, C. In vitro-in vivo correlations for lipophilic, poorly water-soluble drugs. Eur.J.Pharm.Sci. 2000, 11 (Suppl. 2), S73-S80.

17. Dressman, J. B.;Amidon, G. L.; Reppas, C.;Shah, V. P. Dissolution testing as a prognostic tool for oral drug absorption: immediate release dosage forms. Pharm. Res. 1998, 15, 11-20.

18. Galia, E.;Nicolaides, E.;Hörter, D.; Löbenberg, R.; Reppas C.;Dressman J. B. Evaluation of various dissolution media for predicting in vivo performance of Class I and Il drugs. Pharm. Res. 1998, 15,698-705.

19. Blanquet, S.;Zeijdner, E.; Beyssac, E.; Meunier, J-P.; Denis, S.; Havenaar, R.; Alric, M. A dynamic artificial gastrointestinal system for studying the behavior of orally administered drug dosage forms under various physiological conditions. Pharm. Res. 2004,21,37-49.

20. Minekus, M.; Havenaar, R. In vitro model of an in vivo digestive tract. U.S. Patent 5,525,305, June 11,1996.

21. Minekus, M.; Marteau, P.; Havenaar, R.; Hui in't Veld, J. H. A multi compartmental dynamic computer-controlled model simulating the stomach and small intestine. Altern. Lab.Anim. (ATLA) 1995, 23, 197-209.

22. Souliman, S.;Beyssac, E.; Cardot, J. M.;Denis, S.; Alric, M. Investigation of the biopharmaceutical behaviour of theophylline hydrophilic matrix tablets using USP methods and an artificial digestive system. Drug Dev. Ind.Pharm., in press.

23. Souliman, S.;Blanquet, S.;Beyssac, E.; Cardot, J-M. A level $A$ in vitro/in vivo correlation in fasted and fed states using different methods: Applied to solid immediate release oral dosage form. Eur.J.Pharm.Sci. 2006, 27,72-79. 